\title{
NOTES ON LIMIT AND NONLINEAR ELASTIC ANALYSES OF MASONRY ARCHES
}

\author{
Danila Aita ${ }^{1}$, Riccardo Barsotti ${ }^{2}$, Stefano Bennati ${ }^{2}$
}

\begin{abstract}
This contribution aims to be a critical presentation of the studies conducted by the authors over the last ten years on the mechanical response of masonry arches and vaults. More precisely, we focus on a study of masonry arches conducted in parallel via both nonlinear elastic and limit analyses. The onedimensional elastic model for masonry arches incorporates a simple but effective nonlinear constitutive law. In turn, collapse analysis is performed by the so-called 'method of stability areas', originally proposed by Durand-Claye (1867). Rather than offering two alternative paths, the two approaches may be considered two complementary points of view on the same problem.

A reasoned illustration of the concepts in question are furnished by the topics addressed: an analysis of the possible failure mechanisms of a pointed arch subject to its own weight; the search for explicit solutions to the equilibrium problem of a depressed arch subject to a uniformly distributed load; the study of arches of different shapes subject to their own weight and the weight of a superimposed wall.
\end{abstract}

\section{Introduction}

Determining the structural response of masonry arches, vaults and domes, in terms of both displacements and stresses, still represents a challenging task. In fact, a well-known property of masonry structural elements is that their response is typically characterized by a pronounced nonlinear trend even for load intensities that are quite low with respect to the collapse values.

The need to take into account the nonlinear constitutive relations for "masonry" material represents a further challenge that must be faced, except in some special cases. For this reason, especially in the not-so-rare cases of complex building shapes, the solution is sought by recurring to large-dimension numerical analyses, even when geometric nonlinearities are not accounted for (Alfano et al. 2000; Lourenço 2005).

The present work aims to analyze the mechanical response of one of the most common structural elements in masonry constructions: the arch. The two theoretical models that will be used in this regard belong to different, but complementary frameworks, as we shall see: limit analysis, on the one hand, and nonlinear elasticity, on the other hand. Although the two models, which will be illustrated in the first two sections to follow, differ in many respects, they nonetheless share the goal of simplicity as one of their main features. In this regard, it should be noted that the use of simple schemes is motivated not only by reasons of an operational nature, but also by two further considerations regarding the analysis results. First, the simple models generally enable simpler, more concise verification of the results, as they focus attention on the main aspects of the phenomenon in question. Furthermore, to be used effectively, the more complex models - which in principle would allow obtaining more accurate results in closer agreement with experiment - often require knowing a large number of mechanical and geometrical parameters, which are generally uncertain and difficult to determine experimentally. One example that effectively illustrates the many complexities involved in the analysis of masonry structures is offered by the basic, and apparently easy-to-solve, case of a masonry arch subjected to its own weight and the weight of a superimposed wall. Determining the mechanical response of such a system represents a challenging problem due to at least three aspects: the strong material nonlinearities typical of the complex mechanical behavior of masonry, the

\footnotetext{
${ }^{1}$ Department of Architectural Sciences, University of Genoa, Italy

2 Department of Civil and Industrial Engineering, University of Pisa, Italy
} 
pronounced heterogeneity of the masonry, and the large number of geometrical and mechanical parameters involved, which include shape, span and thickness of the arch, the strength of the masonry (usually accounted for by considering some approximate or average values), the inclination of the wall extrados, the considered boundary conditions on the walls' vertical sides and at the arch springings, as well as others. In particular, determining the actual load distribution transmitted from the wall to the arch is by no means a trivial matter, and approximate solutions obtained via finite-element computation codes or, alternatively, by means of elementary calculation schemes, are often considered acceptable in the literature (see, for instance, Cavicchi and Gambarotta, 2005).

In this paper, the two simplified methods that will be used to obtain the solution to some equilibrium problems for a masonry arch will be indicated as the "stability areas method" and the "nonlinear elastic model", respectively. The first solution technique is based on an expressly developed extension of the historical method of "stability areas" introduced by Durand-Claye (1867). It aims at determining the set of statically admissible solutions within the limits imposed by the ultimate compressive and tensile strengths and the limited shear capacity of the joints. When the extent of the "stability area" goes to zero, a limit equilibrium condition is attained for the whole system (Foce and Aita 2003; Aita et al. 2004; 2007). This methodology preserves the fundamental concepts of limit analysis and, at the same time, embodies some aspects of the nonlinear elastic analysis by imposing a restriction on the stress level.

The second solution technique instead focuses on the stress and strain fields generated in the arch, which is considered as a one-dimensional element made of a material offering poor resistance to tension. Such mechanical behavior can be modeled, as a first approximation, via a nonlinear elastic constitutive relation. The problem is tackled by studying and numerically integrating systems of nonlinear equations (Barsotti and Bennati 2001). The condition of incipient collapse is considered to be reached when the residual stiffness of the system falls below a predetermined fraction of its initial value. From a historical point of view, this approach has his roots in the contribution of Signorini, who first proposed direct-method studies of the mechanical behavior of elastic materials unable to bear tensile stresses (Signorini 1925a, 1925b).

The two methods achieve complementary purposes: the stability area method allows for readily determining a collapse load value, while the nonlinear elastic analysis provides a helpful and, in some aspects, essential check of its mechanical significance by following the evolution of the displacement field and extension of the nonlinear regions where cracking and crushing phenomena arise as the load increases.

In the following, the two theoretical frameworks introduced above are used in the search for the solution to three problems, which are described in the third and last section. All three cases presented, which are a selection of case studies examined over the past ten years, refer to plane arches subject to in-plane vertical loads.

In each of the two first applications the attention is focused on one of the two methods in order to highlight its main aspects. In particular, the first case illustrates application of the method of "stability areas" to the collapse of pointed arches, while also taking into account the limited shear strength of the masonry. The second case shows how nonlinear elastic analysis enables obtaining the explicit solution in terms of displacements, stresses, strains and extension of the nonlinear zones in which cracks and damage are expected in the masonry in the case of depressed arches subjected to uniformly distributed vertical loads. Finally, the parallel use of both analysis methods is illustrated in the last case, in which we examine the mechanical system consisting of an arch and a superimposed wall. The problem, which commonly occurs in masonry buildings and bridges, is investigated for arches of different shapes: pointed, semicircular and elliptical. 


\section{The Durand-Claye method}

In 1867, Durand-Claye introduced a graphical procedure aimed at assessing whether admissible thrust's values at the crown section of an arch could be determined for symmetric equilibrium problems. The object of the procedure, which is now commonly indicated as the Durand-Claye method, is to draw a particular plane figure named the stability area. Each point belonging to such area is the extreme of a vector representing an admissible value for the crown thrust, i.e. a thrust that allows for verifying the equilibrium of any given part of the arch and is at the same time compatible with the masonry strength. It is worth observing that here the method proposed by Durand-Claye has been suitably modified so as to account for a nonlinear stress distribution both in tension and compression. In the following we briefly describe the thuslymodified method (a more detailed description is given in Aita et al., 2004).

We consider a symmetric masonry arch. We indicate with $\sigma_{c}$ and $\sigma_{t}$ the masonry compressive and tensile strength, respectively. We assume, for the sake of simplicity, that the arch is loaded by its own weight only.

We begin by examining the ideal voussoir comprised between the crown joint $c_{0} d_{0}$ and a generic joint $c_{i} d_{i}$ (Figure 1). We indicate with $W$ the weight of the voussoir, with $N_{i}$ the normal force at joint $c_{i} d_{i}$, with $P$ the thrust at the crown section, and with $e_{0}$ and $e_{i}$ the eccentricity of their corresponding application points.

If we set the value for the crown eccentricity, $e_{0}$, a first limitation for the thrust value at the crown section, $P$, can be obtained by requiring it to be compatible with the masonry compressive and tensile strength. Unlike the original version of the stability area method, here, any given cross-section of the arch may be subdivided into three different parts: two parts where the normal stress is constant and equal to $\sigma_{c}$ and $\sigma_{t}$, respectively, and a third part where the stress varies linearly between these two threshold values. By varying $e_{0}$, simple calculations allow for drawing the corresponding $c_{0} \omega_{0} d_{0}$ curve, bounding the admissible region. Analogously, by considering the axial force transmitted across the $c_{i} d_{i}$ joint, $N_{i}$, the corresponding $c_{i} \omega_{i} d_{i}$ curve is obtained.

By imposing rotational equilibrium on the voussoir, further limitations are introduced. In fact, it is an easy matter to show that, when the extreme of the vector representing the axial force, $N_{i}$, moves along the $c_{i} \omega_{i} d_{i}$ curve, the corresponding extreme of the thrust at the crown belongs to the hyperbolas $\alpha_{i}$ and $\beta_{i}$.

The last limitation follows directly from the voussoir translational equilibrium and adoption of Coulomb's friction law. The latter requires the magnitude of the shear force transmitted along the $c_{j} d_{j}$ joint not to exceed the limit value $N_{i} \tan \phi$, where $\phi$ is the internal friction angle. In turn, from the translational equilibrium in the direction normal to the $c_{i} d_{i}$ joint, it is straightforward to conclude that two limit values for the horizontal thrusts at the crown section can be assessed, corresponding to the limit inward and outward shear forces, respectively. The two vertical red lines drawn in Figure 1, labeled $\gamma_{i}^{-}$and $\gamma_{i}^{+}$, correspond to these two thrust limit values.

The admissible thrusts $P$ with respect to both the voussoir equilibrium and masonry strength are then represented by the horizontal vectors whose extremes are contained within the area $r_{i} s_{i} p_{i} q_{i}$, internal to the $c_{0} \omega_{0} d_{0}$ curve, and comprised between the hyperbolas $\alpha_{i}$ and $\beta_{i}$, and the straight lines $\overline{\gamma_{i}}$ and $\gamma_{i}^{+}$. 


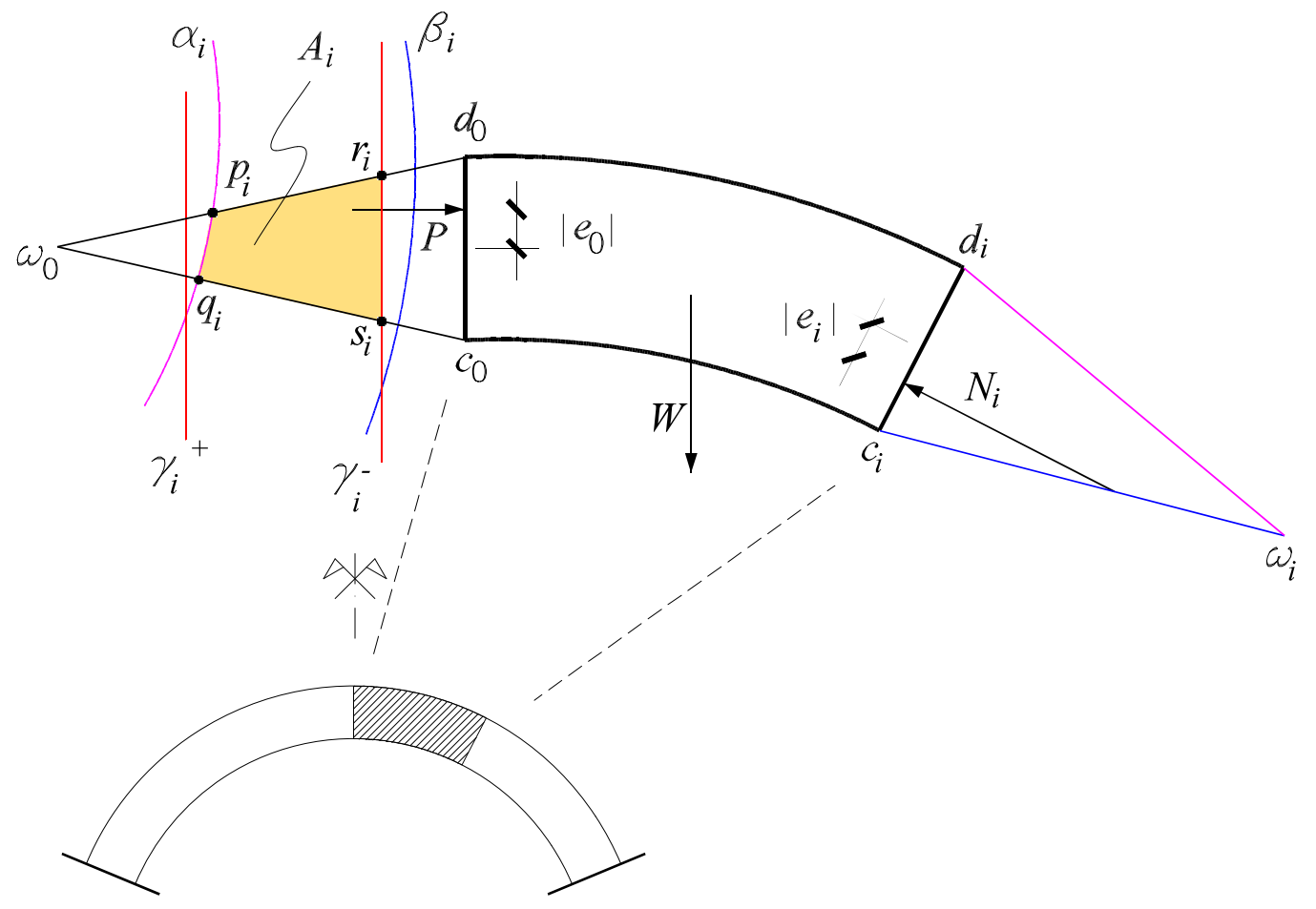

Figure 1 . The stability area $\left(\sigma_{t}=0\right)$

By repeating the foregoing for every joint $i$, the stability area, $A$, common to all the areas $A_{i}$ can be determined. If $A$ has a finite extension, there are infinite admissible thrust values (and infinite corresponding eccentricities), ranging within a suitable interval. On the contrary, if $A$ is empty, no admissible thrust value exists. Finally, as we shall see later, when the stability area $A$ shrinks to a point or to a segment, the limit condition is attained, and a unique admissible thrust value exists.

\section{The non-linear one-dimensional elastic model}

In order to build a simple but effective model, we consider the masonry arch as a deformable curved beam. As usual in the theory of the bending of beams, the cross-section of an arch of height $h$ and, for the sake of simplicity, unit width is assumed to remain plane and normal to the longitudinal fibers after bending, thus neglecting any shear strain. Moreover, the longitudinal normal stresses $\sigma_{\theta}$ are assumed to depend on the corresponding strains $\varepsilon_{\theta}$ according to the same relation holding in a uniaxial state of stress.

The longitudinal strain is linear over any given cross-section. Thus, the kinematics of the arch can be described by three functions of the curvilinear abscissa $s$ along the line of axis, namely: the displacement components $u$ and $v$ of the points of the axis of the arch in the tangential and radial directions, and the rotation $\varphi$ of the cross-section, positive if clock-wise (Figure 2).

Simple calculations, omitted here for the sake of brevity, show that, in the case the arch is circular with radius $R$, the radial displacement $v(\theta)$ is a solution to the differential equation

$$
v^{\prime \prime}+v=-R^{2} \chi-R \varepsilon,
$$


where $\varepsilon$ and $\chi$ are the axial strain and curvature of the line of axis, respectively, $\theta=s / R+\theta_{0}$, the prime denotes differentiation with respect to $\theta$, and $\theta_{0}$ is the starting angle of the circular arc. The integral of eqn. (1) is

$$
\begin{aligned}
v(\theta) & =\left(R \varphi_{0}-u_{0}\right) \sin \left(\theta-\theta_{0}\right)+v_{0} \cos \left(\theta-\theta_{0}\right)+ \\
& -\int_{\theta_{0}}^{\theta} \sin (\theta-t)\left(R^{2} \chi(t)+R \varepsilon(t)\right) d t .
\end{aligned}
$$

In turn,

$$
\varphi(\theta)=\varphi_{0}-\int_{\theta_{0}}^{\theta} R \chi(t) d t,
$$

and

$$
\begin{aligned}
& u(\theta)=-v^{\prime}+R \varphi=u_{0} \cos \left(\theta-\theta_{0}\right)+v_{0} \sin \left(\theta-\theta_{0}\right)+R \varphi_{0}\left(1-\cos \left(\theta-\theta_{0}\right)\right)+ \\
& +\int_{\theta_{0}}^{\theta}\left[\cos (\theta-t)\left(R^{2} \chi(t)+R \varepsilon(t)\right)-R^{2} \chi(t)\right] d t
\end{aligned}
$$

while $u_{0}, v_{0}$ and $\varphi_{0}$ are the tangential and radial components of the displacement and the rotation of the initial cross-section, at $\theta=\theta_{0}$.

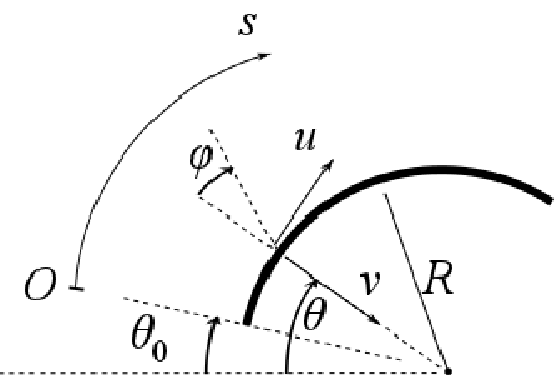

Figure 2. Symbols and notations adopted in the one-dimensional model.

Following Signorini's idea, we adopt for the masonry the piecewise-linear constitutive relation between the longitudinal strain, $\varepsilon_{\theta}$, and stress, $\sigma_{\theta}$, showed in Figure 3. 


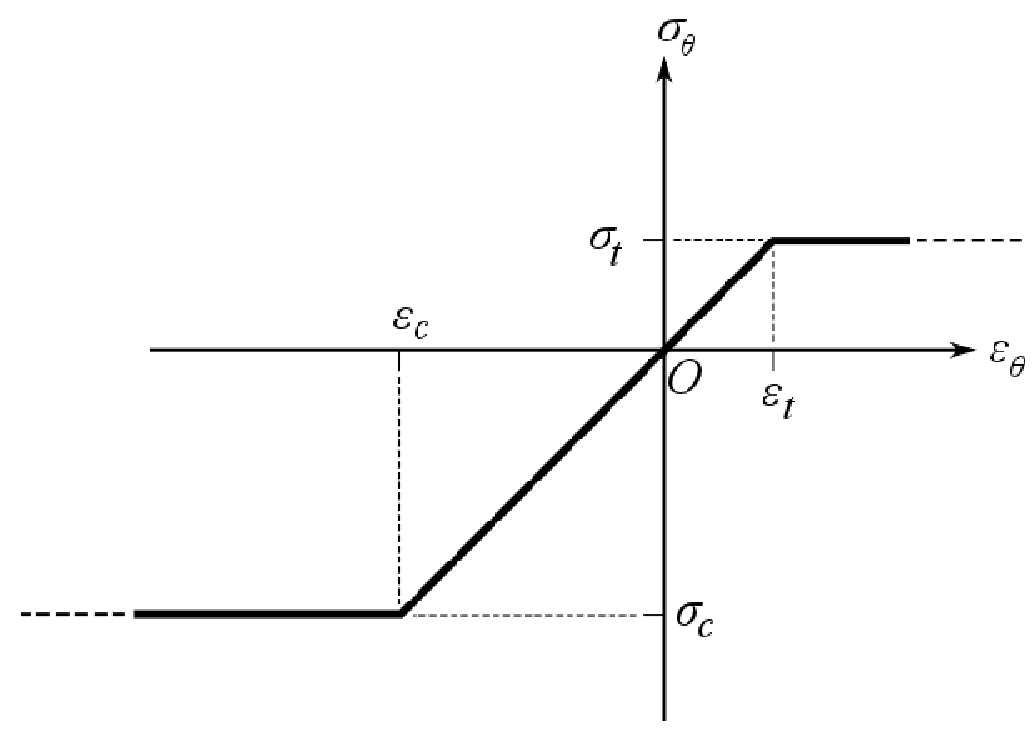

Figure 3. The $\sigma_{\theta}-\varepsilon_{\theta}$ relation.

The simple elastic stress-strain constitutive relation used here reduces to the basic linear case for stresses comprised within the threshold values $\sigma_{c}$ and $\sigma_{t}$, denoting the material's resistance to compression and tension, respectively. Outside this range, strain may grow at constant stress. Consequently, denoting by $E$ the material's Young's modulus, we have

$$
\sigma_{\theta}= \begin{cases}\sigma_{c} & \varepsilon_{\theta} \leq \varepsilon_{c}, \\ E \varepsilon_{\theta} & \varepsilon_{c}<\varepsilon_{\theta}<\varepsilon_{t}, \\ \sigma_{t} & \varepsilon_{\theta} \geq \varepsilon_{t},\end{cases}
$$

where $\varepsilon_{t}=\sigma_{t} / E$ and $\varepsilon_{c}=\sigma_{c} / E$. This simple nonlinear equation, already adopted by the authors in previous works (Bennati and Barsotti, 2001; Aita et al. 2003; 2009; 2012), enables accounting for masonry's weak tensile strength and bounded compressive strength. One drawback is that, in the case $\sigma_{t} \neq 0$, the material is unrealistically assumed to be able to transmit low tensile stresses, even in the presence of high strains.

From constitutive equation (5), and by using the kinematical assumption made on the crosssection, we can build the set of corresponding nonlinear constitutive equations that hold at the cross-sectional level between the kinematic parameters, axial strain $\varepsilon$ and curvature $\chi$, and the internal actions, axial force $N$ and bending moment $M$. Figure 4 shows a plot of the elastic domain in the $(n, m)$ plane, where $n=N / \sigma_{c} h$ and $m=-M / \sigma_{c} h^{2}$ indicate the dimensionless axial force and bending moment, and $t=\sigma_{t} / \sigma_{c}$ the masonry tensile to compressive strength ratio. 


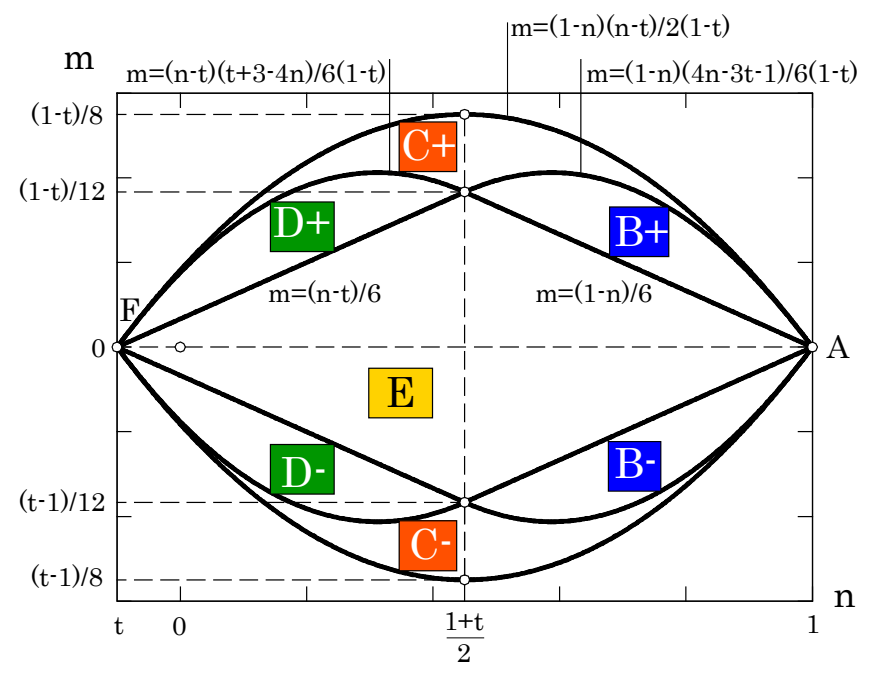

Figure 4. The elastic domain for the arch cross-section.

In each of the seven regions of the elastic domain, the axial strain $\varepsilon$ and the curvature $\chi$ are known functions of the dimensionless axial force and bending moment, $n$ and $m$. By way of example, the constitutive relations between generalized strains and dimensionless internal actions holding in the $\mathrm{B}^{+}$region are

$$
e=\frac{\varepsilon}{\varepsilon_{c}}=1+\frac{8(n-1)^{2}(n-1+3 m)}{9(n-1+2 m)^{2}}, \quad c=\frac{\chi h}{\varepsilon_{c}}=\frac{8(n-1)^{3}}{9(n-1+2 m)^{2}},
$$

or, in inverse form,

$$
n=\frac{e+1}{2}+\frac{c}{8}+\frac{(e-1)^{2}}{2 c}, \quad m=\frac{1-e}{2}-\frac{c}{24}+\frac{(e-1)^{3}}{6 c^{2}} .
$$

Analogous relations, omitted here for the sake of brevity, hold in the other regions of the elastic domain.

Except for very special cases, the equilibrium problem for the arch is statically indeterminate. Thus, the sequence of linear and nonlinear regions along the arch is not known a priori, and the set formed by the nonlinear kinematic equations (2) - (4), the constitutive relations (6) and the equilibrium equations is usually solved numerically through an iterative procedure (Bennati and Barsotti, 1999; 2001). However, for simple cases, such as uniformly loaded flat or depressed arches, an explicit solution can be found, as will be shown in one of the following examples (Aita et al. 2003; 2004).

\section{Some case studies}

The two different solution methods described in the previous sections, namely, the method of stability areas, on the one hand, and nonlinear elastic analysis, on the other, are used to solve the three equilibrium problems illustrated in this third and last section. The applications clearly show that the two methods, though independent, prove to be fruitful when applied complementarily to 
each other. On the one hand, thanks to its simplicity of use, the method of stability areas allows for relatively quick determination of the value of the collapse load, while nonlinear elastic analysis allows for following the evolution of displacements, stresses, and extension of nonlinear regions, and hence evaluating the actual conditions of an arch that is subjected to an assigned distribution of loads.

Some of the main distinctive features of each of the two analysis methods are examined in the first two applications. In particular, an illustrative example application of the stability areas method is shown in the first case, which addresses pointed arches. The method allows for taking into account the influence of masonry's limited shear strength on arch collapse. The case dealt with, in which the arch is subject to its own weight alone, reveals the influence of the thickness and the friction coefficient on arch collapse.

The second case examines the nonlinear elastic analysis of masonry arches, modeled as onedimensional elements. This example aims to demonstrate that it is possible to obtain an explicit solution in terms of displacements, stresses and extension of the regions in which the behavior is nonlinear, where the onset and progression of cracking and material damage would be expected in the case of greatly depressed arches subject to uniformly distributed vertical loads. The analysis is conducted by assuming an increasing loading process, so as to describe the evolution of the solution up to collapse.

Lastly, the parallel use of both analysis methods is illustrated in the last of the three cases, in which the mechanical system formed by an arch and an overlying wall is considered. The problem, which occurs commonly in masonry buildings and bridges, has been examined for arches of different shapes: pointed, circular and elliptical. The main objective is to determine the stress levels as a function of the main geometrical and mechanical parameters, and thereby assess the safety margin under conditions of incipient collapse, as well as the actual mechanism by which such collapse would occur (Aita, Barsotti and Bennati 2012).

\subsection{Pointed arches under their own weight}

The "stability areas" method, suitably modified to account for masonry's limited compressive strength, allows for easily determining the limit values for the thrust corresponding to the attainment of some limit condition along a generic $c_{i} d_{i}$ joint by considering the corresponding stability area, $A_{i}$. As already described in Section 2, when the extreme point of the crown thrust vector is on the border of $A_{i}$, a limit condition is attained on the $c_{i} d_{i}$ joint (Figure 1). If the bending moment at the $c_{i} d_{i}$ joint reaches the limit value (which in turn depends on the eccentricity and magnitude of the axial force), the corresponding limit condition concerns the rotational equilibrium of the voussoir between the crown and the joint in question. If, instead, the shear force at the $c_{i} d_{i}$ joint reaches the limit value, a limit condition for the translational equilibrium of the same voussoir ensues. By scanning every joint along the arch, we determine the arch's overall stability area $A$ as the intersection of all $A_{i}$ areas.

If $A$ is empty, equilibrium of the arch is not possible. In other words, the internal forces are not statically admissible. If the area $A$ is greater than zero, there are (infinite) values of the eccentricity and magnitude of $P$ that correspond to statically admissible internal force distributions. Lastly, the limit case is when $A$ shrinks to zero: as we shall see later, when A becomes a segment, only one statically admissible value of the magnitude of $P$ can be found, while in the case that $A$ becomes a single point, only one statically admissible value of both the eccentricity and magnitude can be found. In these latter cases, the arch is in a limit condition.

By varying both the arch thickness, $h$, and friction coefficient, $\mu$, the Durand-Claye method enables finding the pairs $(h, \mu)$ that correspond to a limit condition for the arch. In each of these 
cases, the limit value of the eccentricity and/or magnitude of $P$ is determined. It is worth noting that, although masonry is considered here as a non-standard material, we can nevertheless find the collapse mechanism that may actually arise in correspondence to each limit thrust value (some further considerations on this can be found in Sinopoli et al. 2007). For any given collapse mechanism, each portion of the arch between any two consecutive joints that attain a limit condition will undergo rigid motion. We classify the different types of rigid motion, together with the corresponding symbols adopted for the crown thrust, in the following Tables 1 and 2, where the centers of rotation and the application points of the thrust at the crown are defined assuming infinite compressive strength, $\sigma_{c}$; in the case of limited $\sigma_{c}$, the terms "intrados", "extrados" are to be interpreted as "near the intrados" and "near the extrados", while a limit condition is reached at the crown or at joint $c_{i} d_{i}$.

\begin{tabular}{lll}
\hline $\begin{array}{l}\text { Centre of rotation } \\
\text { at joint } c_{i} d_{i}\end{array}$ & $\begin{array}{l}\text { Application point of } \\
\text { thrust at crown }\end{array}$ & Thrust value \\
\hline \multirow{4}{*}{ Intrados } & Extrados & $P_{\min }^{r, e}\left(\theta_{i}\right)$ \\
& Intrados & $P_{\min }^{r, i}\left(\theta_{i}\right)$ \\
& Internal & $P_{\min }^{r, *}\left(\theta_{i}\right)$ \\
\hline \multirow{3}{*}{ Extrados } & Extrados & $P_{\max }^{r, e}\left(\theta_{i}\right)$ \\
& Intrados & $P_{\max }^{r, i}\left(\theta_{i}\right)$ \\
& Internal & $P_{\max }^{r, *}\left(\theta_{i}\right)$
\end{tabular}

$\overline{\text { Table 1. Notation for limit thrust values corresponding to hinging mechanisms. }}$

\begin{tabular}{l|ll}
\hline & Direction & Thrust value \\
\hline \multirow{3}{*}{ Relative sliding } & Downwards & $P_{\min }^{s}\left(\theta_{i}\right)$ \\
& Upwards & $P_{\max }^{s}\left(\theta_{i}\right)$ \\
\hline
\end{tabular}

Table 2. Notation for limit thrust values corresponding to sliding mechanisms.

By setting $l=R=10 \mathrm{~m}, \gamma=20 \mathrm{kN} / \mathrm{m}^{3}, \sigma_{c}=-20 \mathrm{MPa}, \sigma_{t}=0$, and varying the thickness $h$ and friction coefficient $\mu$, different collapse modes for the pointed arch can be found. These have been identified and classified according to the well-known eight collapse modes of a symmetric arch illustrated by Michon in 1857. The results, reported in the following, are taken from (Aita et al., 2004; 2007).

\section{$\underline{\text { Sliding collapse (Modes } 5 \text { and } 6 \text { ) }}$}

When the stability area reduces to a vertical line, the range of admissible thrusts shrinks to a single value and collapse can occur by Mode 5 or Mode 6 (Figure 5). In particular, it is an easy matter to verify that Mode 5 (Figure 5a) can occur when the condition 


$$
\begin{aligned}
& P=\max P_{\min }^{s}(\theta)=P_{\min }^{s}\left(\theta_{i}\right)= \\
& =\min P_{\max }^{s}(\theta)=P_{\max }^{s}\left(90^{\circ}\right),
\end{aligned}
$$

for the crown thrust is fulfilled. The angle value $\theta_{i}$, corresponding to the joint between the crown and the abutment where the shear force attains a limit condition, depends on the particular values chosen for $h$ and $\mu$. Condition (8) is verified for:

$$
h>128 \mathrm{~cm} \text { and } \mu \leq 0.19 \text {. }
$$

Analogously, Mode 6 (Figure 5b) can occur when:

$$
\begin{aligned}
& P=\min P_{\max }^{s}(\theta)=P_{\max }^{s}\left(\theta_{i}\right)= \\
& =\max P_{\min }^{s}(\theta)=P_{\min }^{s}\left(\theta_{j}\right) \quad \text { with } \theta_{i}<\theta_{j},
\end{aligned}
$$

Once again, the angle values $\theta_{i}$ and $\theta_{j}$, corresponding to the joints where the shear force attains a limit condition, depend on the particular values chosen for $h$ and $\mu$. Condition (9) is verified for

$$
86 \mathrm{~cm}<h<128 \mathrm{~cm} \text { and } \mu=\mu_{6}(h),
$$

where $\mu_{6}$ takes values in the range of $0.19<\mu_{6}<0.21$.

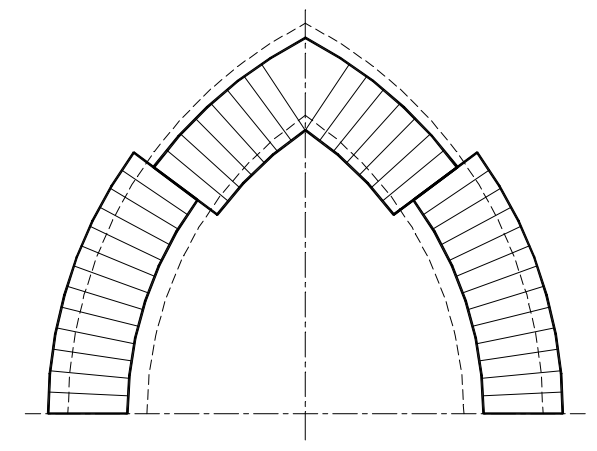

(a) Mode 5

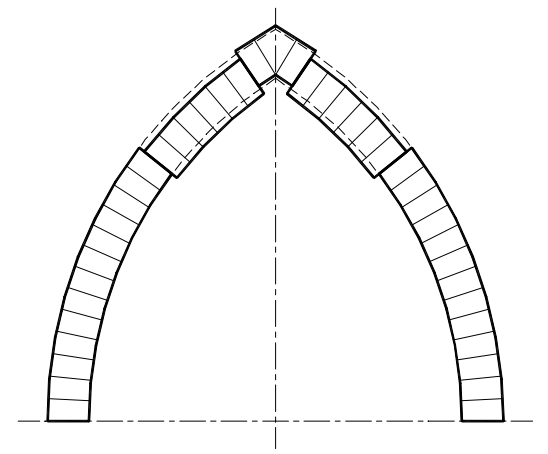

(b) Mode 6

Figure 5. Sliding collapse mechanisms (modes 5 and 6).

The transition between modes 5 and 6 takes place for $\mu=0.19$ and $h=128 \mathrm{~cm}$ (Figure $6 \mathrm{~b}$ ). Such limit condition corresponds to

$$
P=P_{\max }^{s}\left(35.2^{\circ}\right)=P_{\min }^{s}\left(52.5^{\circ}\right)=P_{\max }^{s}\left(90^{\circ}\right)=0.5 \mathrm{kN} .
$$

In the $\left(P, e_{0}\right)$ plane, the corresponding stability area is the vertical orange line shown in Figure 6 a. 


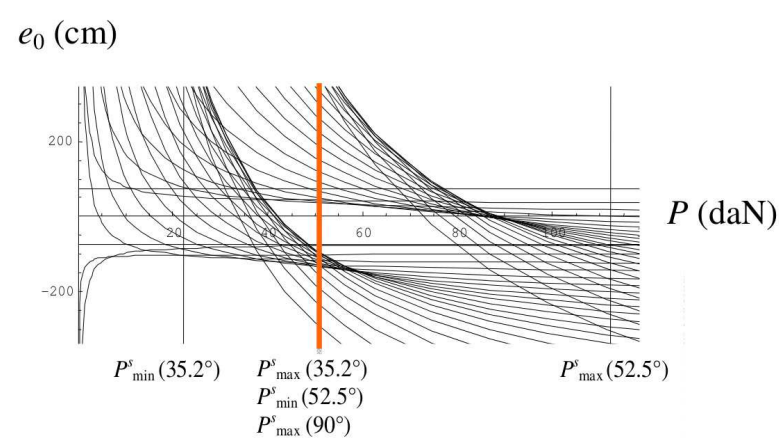

(a)

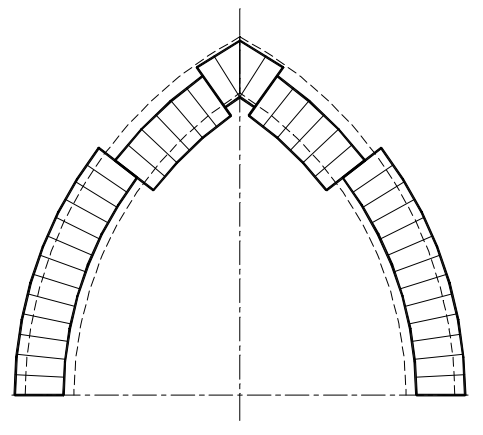

(b)

Figure 6. Transitional collapse 5 - 6;

(a) stability area in the $\left(P, e_{0}\right)$ plane, $(b)$ scheme of the collapse mechanism.

\section{Mixed-mode collapse (Mode 4*)}

For any given thickness value in the range of $40 \mathrm{~cm}<\mathrm{h}<86 \mathrm{~cm}$, the stability area reduces to a single point if the friction coefficient equals a limit threshold $\mu_{4}$, whose values depend on $h$ and fall within the range of $0.21<\mu_{4}<0.33$. In such cases, by referring to the $\left(P, e_{0}\right)$ plane, the straight line corresponding to the limit thrust for the translational equilibrium at some joint $c_{i} d_{i}$ and the two curves corresponding to limit rotational equilibrium at two other joints $c_{j} d_{j}$ and $c_{k} d_{k}$ intersect each other at the same point (Figure 7a), the mixed sliding-hinging collapse mode schematized in Figure $7 \mathrm{~b}$ can occur, and the limit thrust condition is:

$$
P=\min P_{\max }^{s}(\theta)=P_{\max }^{s}\left(\theta_{i}\right)=P_{\max }^{r * *}\left(\theta_{j}\right)=P_{\min }^{r * *}\left(\theta_{k}\right), \quad \text { with } \theta_{i}<\theta_{j}<\theta_{k} .
$$

We indicate this collapse mode as Mode 4*, because it is somewhat similar to Michon's Mode 4 , according to which, however, the limit shear joint should be located between the two joints where a rotational limit condition is reached.

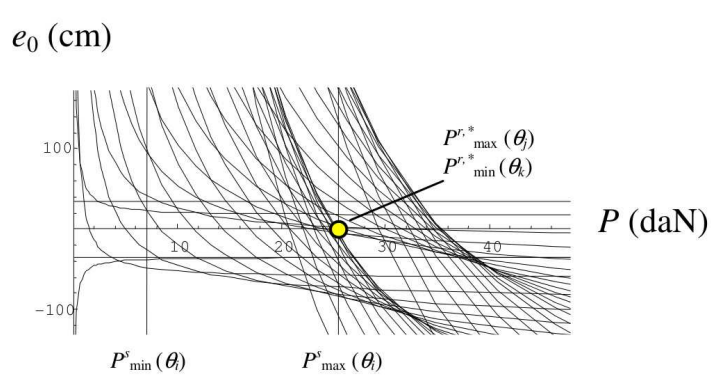

(a)

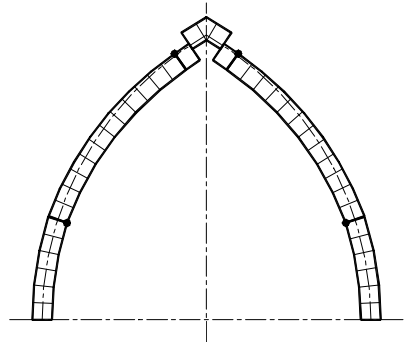

(b)

Figure 7. Mixed sliding-hinging collapse mode $4 *$;

(a) stability area in the $\left(P, e_{0}\right)$ plane, $(b)$ scheme of the collapse mechanism.

By decreasing the thickness down to $h=86 \mathrm{~cm}$, it can be seen that for $\mu=0.21$ the two curves corresponding to limit rotational equilibrium at joint $\theta=37.4^{\circ}$ and $\theta=69.5^{\circ}$ and the 
straight line corresponding to the limit thrust for the translational equilibrium (Mode 6, with $\theta_{i}=$ $34.4^{\circ}$ and $\theta_{j}=52^{\circ}$ ) all intersect each other at the same point (the yellow circle in Figure 8a). In this case, the stability area shrinks to that point, and the transitional mixed sliding-hinging collapse mode illustrated in Figure 8b may occur. Such limit condition corresponds to

$$
P=P_{\max }^{s}\left(34.4^{\circ}\right)=P_{\max }^{r, *}\left(37.4^{\circ}\right)=P_{\min }^{s}\left(52^{\circ}\right)=P_{\min }^{r, *}\left(69.5^{\circ}\right)=0.32 \mathrm{kN} \text {. }
$$

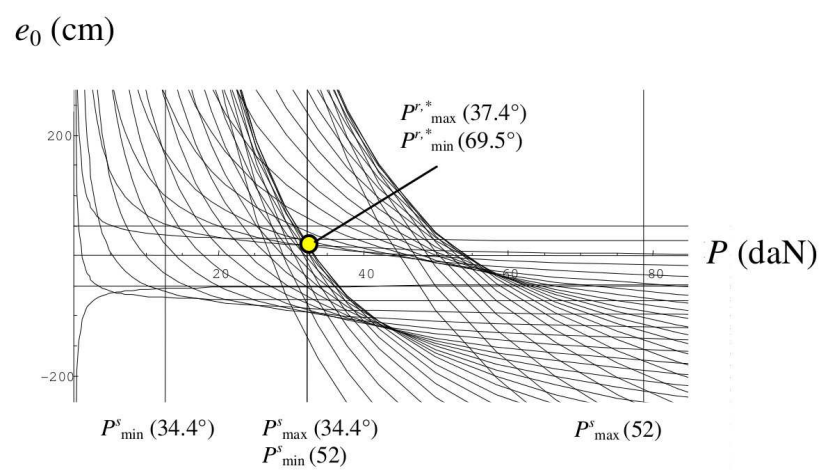

(a)

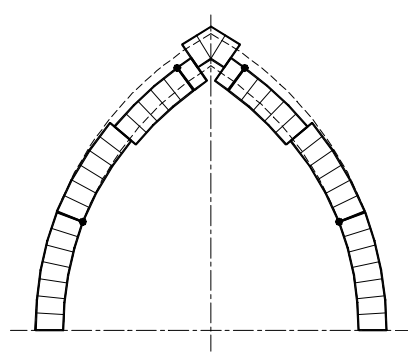

(b)

Figure 8. Transitional mixed-mode collapse 6 - 4*;

(a) stability area in the $\left(P, e_{0}\right)$ plane, $(b)$ scheme of the collapse mechanism.

\section{Hinging collapse (Mode 2)}

For $h=40 \mathrm{~cm}$ and $\mu>0.33$ the stability area reduces to a single point (Figure 9a), and the hinging collapse mode represented in Figure $9 \mathrm{~b}$ can occur. The corresponding limit condition becomes

$$
\begin{gathered}
P=\min P_{\max }^{r, i}(\theta)=P_{\max }^{r, i}\left(39.6^{\circ}\right)= \\
=\max P_{\min }^{r, i}(\theta)=P_{\min }^{r, i}\left(69.2^{\circ}\right)=0.19 \mathrm{kN} .
\end{gathered}
$$

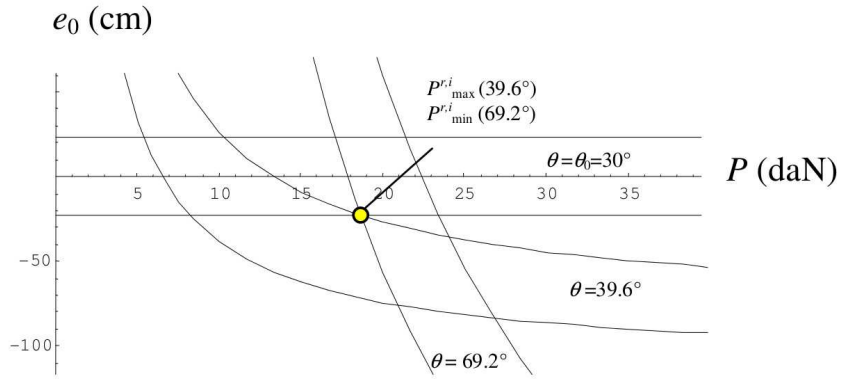

(a)

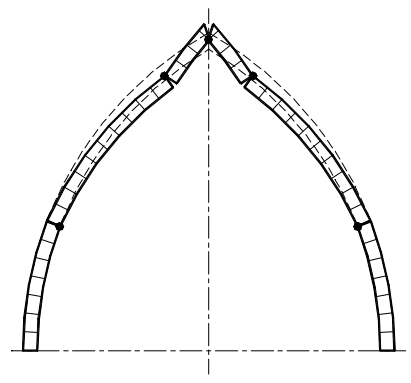

(b)

Figure 9. Hinging collapse (mode 2);

(a) stability area in the $\left(P, e_{0}\right)$ plane, $(b)$ scheme of the collapse mechanism. 
For $h=40 \mathrm{~cm}$ and $\mu=0.33$ the transitional mixed-mode collapse $2-4^{*}$ can arise. The corresponding limit condition is:

$$
\begin{aligned}
& P=\min P_{\max }^{s}(\theta)=P_{\max }^{s}\left(33.6^{\circ}\right)= \\
& =\min P_{\max }^{r, i}(\theta)=P_{\max }^{r, i}\left(39.6^{\circ}\right)= \\
& =\max P_{\min }^{r, i}(\theta)=P_{\min }^{r, i}\left(69.2^{\circ}\right)=0.19 \mathrm{kN}
\end{aligned}
$$

The foregoing results are summarized in Figure 10 in terms of thickness and friction coefficient. Each segment along the 'safe' domain border corresponds to one of the collapse modes illustrated above; the transitional collapse mechanisms correspond to points $\mathrm{A}, \mathrm{B}, \mathrm{C}$.

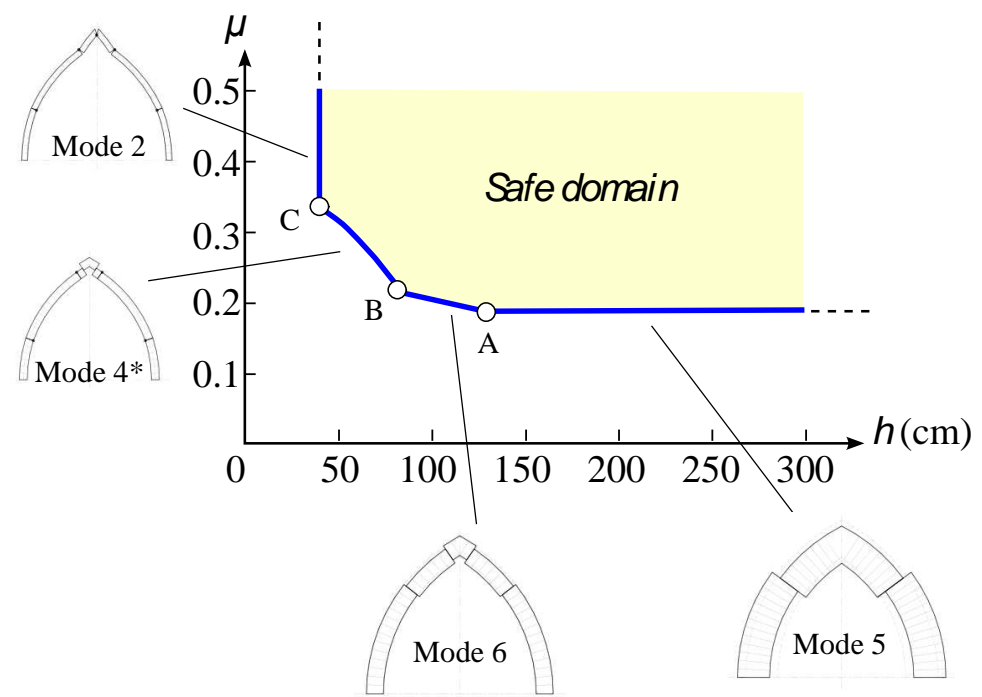

Figure 10. Collapse modes in the $(h, \mu)$ plane.

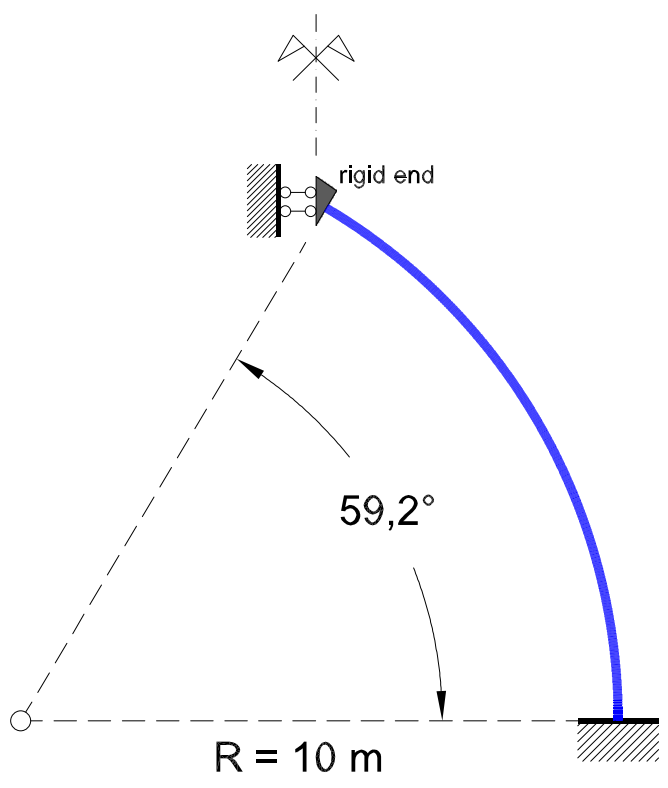

(a)

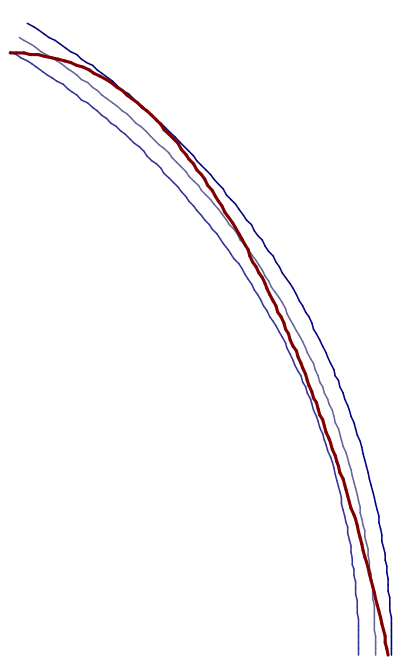

(b)

Figure 11. (a) Structural scheme; (b) line of thrust. 
The nonlinear elastic model of the arch can be used to deal with the same problem already studied using the Durand-Claye method. Although comparison of the two solutions is necessarily incomplete, as the elastic model in its current version neglects shear deformability, it is worthwhile underlining that the results of the nonlinear elastic analysis are in good agreement with those obtained with the stability areas method. This is particularly evident for the limit case corresponding to a pure flexural collapse mechanism (very thin arch). Some correspondences are also evident between the two solution methods in the other limit case (very thick arch), in which a pure shear collapse mechanism takes place.

The nonlinear elastic model has been applied to the structural scheme shown in Figure 11a, and the equilibrium problem solved for different thickness values, ranging from $45 \mathrm{~cm}$ to 150 $\mathrm{cm}$. For a thin arch, the position of the line of thrust (Figure 11b) reveals that the arch attains a limit condition that can be considered near collapse. Such a situation is confirmed by the diagram of the normal stresses at the arch's extrados and intrados (Figure 12a), characterized by high compressions. It should be noted that the hinge positions for collapse mode 2 (Figure 9) are fully compatible with the line of thrust in Figure 11.

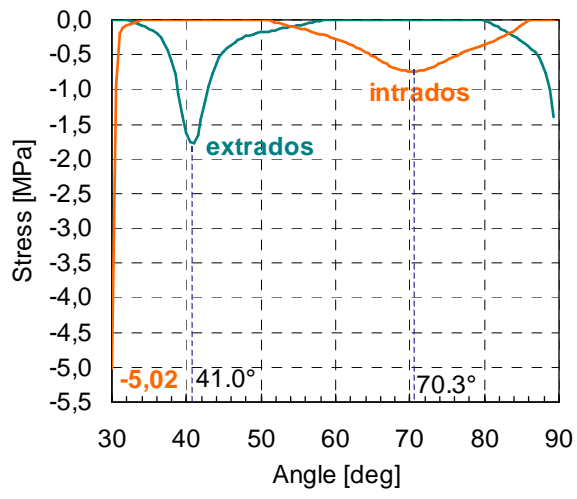

(a)

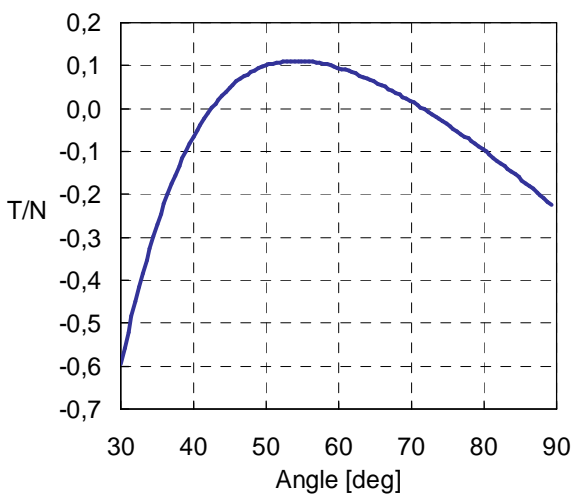

(b)

Figure 12. (a) Normal stress distribution; (b) shear over axial force ratio $(h=45 \mathrm{~cm})$.

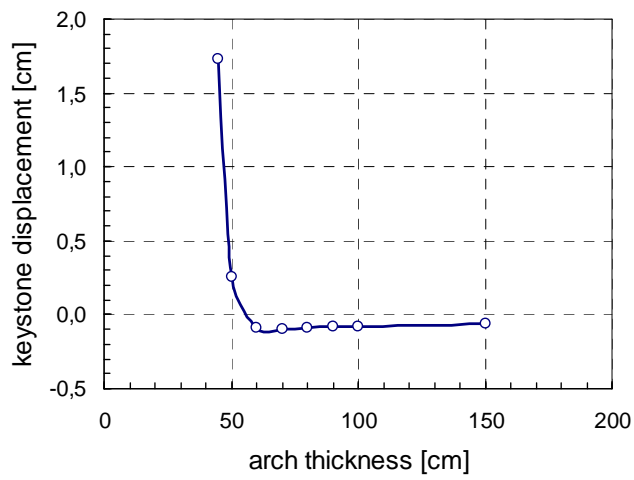

Figure 13. Keystone vertical displacement as a function of arch thickness.

Figure 13 shows the vertical displacement values at the keystone plotted against the arch thickness. The rapid increase in displacement as the thickness approaches $45 \mathrm{~cm}$ once again confirms that in this case the arch is close to a hinging collapse mechanism. It should also be noted that the sign of the displacement for the two cases corresponding to $45 \mathrm{~cm}$ and $150 \mathrm{~cm}$ are consistent with the mechanisms determined via the stability areas method. 


\subsection{A depressed circular arch subjected to a vertical load: some explicit solutions}

Let us consider the problem for the depressed circular arch showed in Figure 14a, for which we indicate with $l, R$ and $2 \alpha$, the clear span, the radius of the line of axis and the center angle of the arch, respectively, and assume by hypothesis that the ratio $l / R$ is small with respect to unity (Aita et al., 2003). Here we will show that tackling a simplified version of the problem enables finding the analytical expressions for the arch's displacements and rotations by making some simple but reasonable hypotheses.

As already pointed out in the foregoing, the equilibrium problem for the arch in question is all but simple to solve because, among other reasons, in general the load distribution that the wall transmits to the arch, as well as the constraints imposed on the arch end sections are all unknown.

We represent the masonry arch as a deformable curved beam. For the sake of simplicity, a uniformly distributed vertical load will model the actions exerted on the arch by the overlying wall. Moreover, we will assume that at both ends the horizontal thrust, $P$, and the couple of moment $M_{B}$ are assigned, while the vertical component of the displacement is fully restrained (Figure 14b). It is worth noting that such a statically determinate problem could be considered a first rough scheme somehow related to the case in which the structures supporting the arch (i.e. piers and abutments) have attained a limit condition. In other words, the end actions, $P$ and $M_{B}$, could be considered limit values for the reactive forces exerted by the piers and abutments.
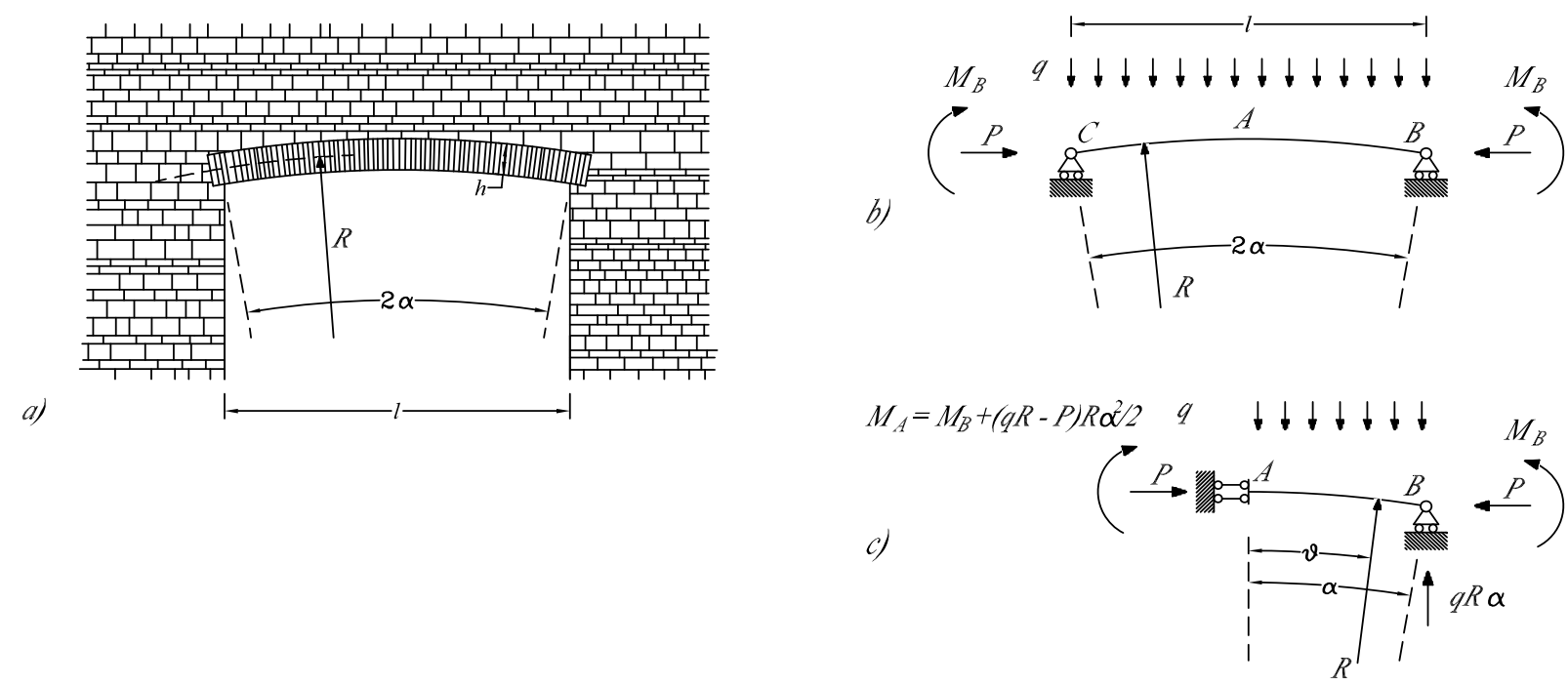

Figure 14. (a) Depressed circular arch loaded by a superimposed wall; $(b)$ the corresponding statically determinate problem; $(c)$ the mechanical scheme.

Symmetry enables limiting our analysis to the right half of the arch (Figure 14c), to which end we indicate $\theta$ (with $0 \leq \theta \leq \alpha$ ) as the angle formed between any given cross-section and the keystone. In order to obtain explicit expressions for the displacements and rotations of the crosssections, it is useful to make some simplifications. Firstly, since the values of $\theta$ are sufficiently small with respect to unity, the expressions for both the axial force and the bending moment will be simplified according to a Taylor series up to the second order. Moreover, by considering that the axial force can, for a sufficiently thin arch, be approximated as a constant and that the thrust is of the same order of magnitude as the resultant of the load, we assume that: 
$n(\theta)=n_{0}, \quad m(\theta)=m_{A}+\frac{\left(n_{0} \eta-p\right)}{2 \eta^{2}} \theta^{2}$,

where we have set $n_{0}=-P / \sigma_{c} h, m_{A}=-M_{A} / \sigma_{c} h^{2}, p=-q / \sigma_{c}$ and $\eta=h / R$.

Since the arch is a statically determinate structure, the internal forces are known, and equations (12) enable establishing the mechanical response (linear elastic, nonlinear in tension, etc.) within each segment of the line of axis. Therefore, at each cross-section we can choose the suitable constitutive relation from those listed in set (6) and obtain the explicit expressions for the cross-section's rotation and displacement by integrating (2)-(4). To this end, we express rotations $\varphi$ and displacements, $u$ and $v$, as

$\varphi(\theta)=\varphi_{0}+F(\theta)$

$v(\theta)=\left(R \varphi_{0}-u_{0}\right) \theta+v_{0}\left(1-\frac{\theta^{2}}{2}\right)+G(\theta)-\theta H(\theta)$,

$u(\theta)=u_{0}\left(1-\frac{\theta^{2}}{2}\right)+v_{0} \theta+\frac{R \varphi_{0} \theta^{2}}{2}-\frac{\theta^{2}}{2} H(\theta)+\theta G(\theta)+L(\theta)$

in which $\theta_{0}=0$ and

$F(\theta)=-\int_{0}^{\theta} R \chi d t$,

$G(\theta)=\int_{0}^{\theta} t\left(R^{2} \chi+R \varepsilon\right) d t$,

$H(\theta)=\int_{0}^{\theta}\left(R^{2} \chi+R \varepsilon\right) d t$,

$L(\theta)=\int_{0}^{\theta}\left[R \varepsilon-\frac{t^{2}}{2}\left(R^{2} \chi+R \varepsilon\right)\right] d t$.

The distributions of the curvature and axial strain, $\chi(\theta)$ and $\varepsilon(\theta)$, can be determined from that of the internal forces, $n(\theta)$ and $m(\theta)$, and (16)-(19) can be integrated, thus yielding the explicit expressions for $F(\theta), G(\theta), H(\theta)$ e $L(\theta)$. Such integrals are easily assessed by subdividing the line of axis into a finite number of segments, each characterized by a different mechanical response (linear elastic, nonlinear in tension, etc.). Lastly, constants $u_{0}, v_{0}$ and $\varphi_{0}$ are determined by imposing the boundary conditions at the arch end sections. Further details can be found in a forthcoming paper (Aita et al., 2015a). 


\begin{tabular}{ll}
\hline Dimensionless parameters & \\
\hline$\eta=h / R=0.02$ & $m_{\mathrm{A}}=-M_{\mathrm{A}} / \sigma_{c} h^{2}=0.045$ \\
$t=\sigma_{t} / \sigma_{c}=-0.015$ & $n_{0}=-P / \sigma_{c} h=0.1$ \\
$\varepsilon_{c}=\sigma_{c} / \mathrm{E}=-2.9 \times 10^{-3}$ & $p=-q / \sigma_{c}=0.006$ \\
$\mathcal{E}_{t}=t \varepsilon_{c}=4.4 \times 10^{-5}$ & \\
\hline
\end{tabular}

Table 3. Dimensionless parameter values.

As an example, let us now consider a $10 \mathrm{~m}$ span, $100 \mathrm{~cm}$ thick depressed arch. The line of axis is a circular arc with radius $R=50 \mathrm{~m}$ and central angle $2 \alpha$ of $0.2 \mathrm{rad}$. For the sake of simplicity, we assume that the arch is of unit width $(1 \mathrm{~m})$ in the transverse direction. A horizontal thrust of $P=2000 \mathrm{kN}$ and a bending moment $M_{\mathrm{B}}=-100 \mathrm{kNm}$ are imposed on both the arch's end sections. A vertical load per unit length of the horizontal projection of the line of axis, $q=120 \mathrm{kN} / \mathrm{m}$, is uniformly distributed throughout the arch (Figure 15). Lastly, we assume that $E=7 \mathrm{GPa}$ is the masonry Young's modulus and that the masonry tensile and compressive strengths are equal to $\sigma_{t}=0.3 \mathrm{MPa}$ and $\sigma_{c}=-20 \mathrm{MPa}$, respectively. The corresponding values for the dimensionless parameters appearing in relations (16)-(19) are listed in Table 3.

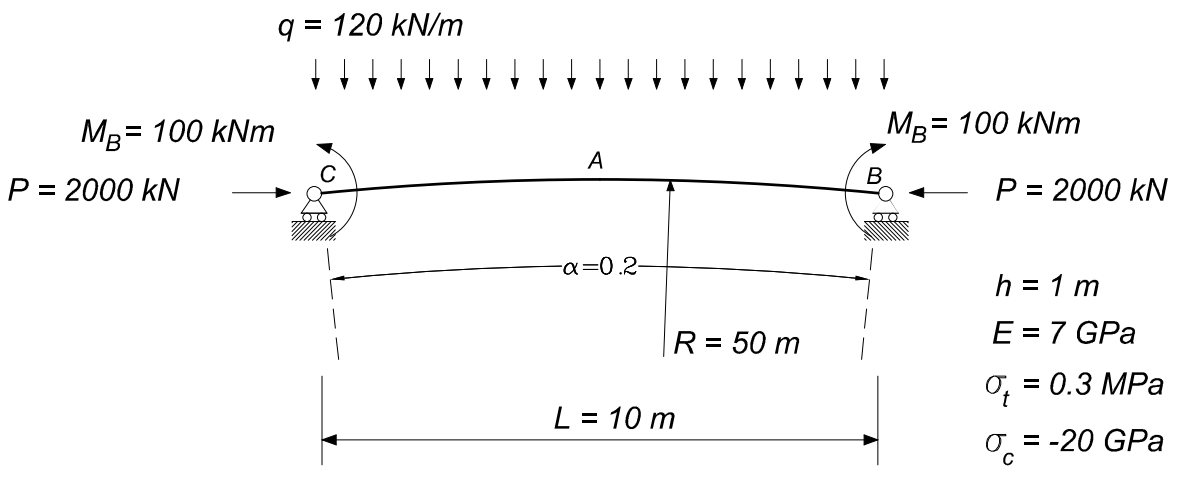

Figure 15. Statically determinate example: mechanical scheme.

As before, due to symmetry we can limit the analysis to the arch's right side alone. Ideally, the arch's line of axis may be subdivided into two parts: the first, which starts at the keystone, is in a mechanical regime that is nonlinear under tension (region D+, Figure 4), while the response in the second part is linear elastic (region $\mathrm{E}$ ). The angle $\theta_{1}$ corresponding to the point separating the arch's D+ and E segments can be obtained via (12). By imposing that $m(\theta)=m_{1}$, we obtain $\theta_{1}=$ $0.072 \mathrm{rad}$. By suitably adapting (13)-(15) to the present case, the following relations are easily obtained:

$$
\begin{aligned}
& \varphi(\theta)= \begin{cases}F^{D+}(\theta), & 0 \leq \theta \leq \theta_{1}, \\
F^{E}(\theta)+F_{1}, & \theta_{1} \leq \theta \leq \alpha,\end{cases} \\
& v(\theta)=v_{0}\left(1-\frac{\theta^{2}}{2}\right)+ \begin{cases}G^{D+}(\theta)-\theta H^{D+}(\theta), & 0 \leq \theta \leq \theta_{1}, \\
G^{E}(\theta)+G_{1}-\theta\left[H^{D+}(\theta)+H_{1}\right], & \theta_{1} \leq \theta \leq \alpha,\end{cases}
\end{aligned}
$$


$u(\theta)=v_{0} \theta+ \begin{cases}L^{D+}(\theta)-\frac{\theta^{2} H^{D+}(\theta)}{2}+\theta G^{D+}(\theta), & 0 \leq \theta \leq \theta_{1}, \\ L^{E}(\theta)+L_{1}-\frac{\theta^{2}\left[H^{E}(\theta)+H_{1}\right]}{2}+\theta\left[G^{E}(\theta)+G_{1}\right], & \theta_{1} \leq \theta \leq \alpha,\end{cases}$

in which

$F_{1}=F^{D+}\left(\theta_{1}\right)-F^{E}\left(\theta_{1}\right), G_{1}=G^{D+}\left(\theta_{1}\right)-G^{E}\left(\theta_{1}\right), H_{1}=H^{D+}\left(\theta_{1}\right)-H^{E}\left(\theta_{1}\right), L_{1}=L^{D+}\left(\theta_{1}\right)-L^{E}\left(\theta_{1}\right)$.

The analytical expressions appearing in (20)-(22), all functions of angle $\theta$, are listed in Table 4 . The constant $v_{0}$ is determined by imposing

$u(\alpha) \sin \alpha+v(\alpha) \cos \alpha=0$,

as the constraint condition at the springing, which yields $v_{0}=42.8 \mathrm{~mm}$.

Region $D+$

$$
\begin{array}{ll}
a=\frac{n_{0} \eta-p}{\eta^{2}\left(t-n_{0}+2 m_{A}\right)}=400 & b=-\frac{3\left(n_{0} \eta-p\right)}{2 \eta^{2}\left(\left(t-n_{0}\right)(\eta-1)+3 m_{A}\right)}=60.56 \\
c=t-n_{0}+3 m_{A}=0.02 & d=\frac{3\left(n_{0} \eta-p\right)}{2 \eta^{2}}=-5 \\
k_{1}=-\frac{4 \varepsilon_{c}\left(t-n_{0}\right)^{3}}{9 \eta\left(t-n_{0}+2 m_{A}\right)^{2}}=-0.155 & k_{2}=-\frac{8 R \varepsilon_{c}\left(t-n_{0}\right)^{2}\left[\left(t-n_{0}\right)(\eta-1)+3 m_{A}\right]}{9 \eta\left(t-n_{0}+2 m_{A}\right)^{2}}=0.266 \\
k_{3}=-\frac{8 R \varepsilon_{c}\left(t-n_{0}\right)^{2}}{9\left(t-n_{0}+2 m_{A}\right)^{2}}=2687 & G^{D+}(\theta)=k_{2}\left(\frac{b}{2 a^{2}} \ln \left(1+a \theta^{2}\right)+\frac{(a-b) \theta^{2}}{2 a\left(1+a \theta^{2}\right)}\right)+R t \varepsilon_{c} \frac{\theta^{2}}{2} \\
F^{D+}(\theta)=k_{1}\left(\frac{\arctan \theta \sqrt{a}}{\sqrt{a}}+\frac{\theta}{1+a \theta^{2}}\right) & H^{D+}(\theta)=k_{2}\left(\frac{(a+b) \arctan \theta \sqrt{a}}{2 a \sqrt{a}}+\frac{\theta(a-b)}{2 a\left(1+a \theta^{2}\right)}\right)+R t \varepsilon_{c} \theta \\
L^{D+}(\theta)=\left(\frac{k_{3}(a c+d)}{2 a^{3 / 2}}-\frac{k_{2}(a-3 b)}{4 a^{5 / 2}}\right) \arctan \theta \sqrt{a}+\left(\frac{k_{3}(a c-d)}{2 a}-\frac{k_{2}(b-a)}{4 a^{2}}\right) \frac{\theta}{1+a \theta^{2}}+ \\
+\left(R t \varepsilon_{c}-\frac{k_{2} b}{2 a^{2}}\right) \theta-\frac{R t \varepsilon_{c}}{6} \theta^{3} \\
\hline
\end{array}
$$

\section{Region E}

$$
\begin{aligned}
& i=-R \varepsilon_{c}\left(\frac{12 m_{A}}{\eta}-n_{0}\right)=3843 \quad j=-\frac{R \varepsilon_{c}\left(n_{0} \eta-p\right)}{\eta^{3}}=-71429 \quad k=R \varepsilon_{c}\left(\frac{2 m_{A}}{\eta}-\frac{n_{0}}{6}\right)=-641 \\
& F^{E}(\theta)=\frac{12 \varepsilon_{c}}{\eta}\left[m_{A} \theta+\frac{\left(n_{0} \eta-p\right)}{6 \eta^{2}} \theta^{3}\right] \quad G^{E}(\theta)=\frac{i}{2} \theta^{2}+\frac{3 j}{2} \theta^{4} \\
& H^{E}(\theta)=i \theta+2 j \theta^{3} \\
& L^{E}(\theta)=R \varepsilon_{c} n_{0} \theta+k \theta^{3}-\frac{3 j}{5} \theta^{5}
\end{aligned}
$$

Table 4. Explicit expressions of the integrals appearing in (20) - (22).

The diagrams of the rotations and displacements of the line of axis are plotted in Figure 16, together with the arch's deformed shape. In the case under examination it is a straightforward matter to verify that the nonlinear response predicted by the model illustrated here differs from that which would be obtained by assuming linear elastic behavior (Figure 16a, b, d). In particular, 
the presence of an arch segment where the behavior is nonlinear modifies the arch's mechanical response so that the displacements and rotations are tripled with respect to the linear elastic solution. Thus, the simple nonlinear model proposed here is in agreement, at least qualitatively, with the experimentally reported arch response.
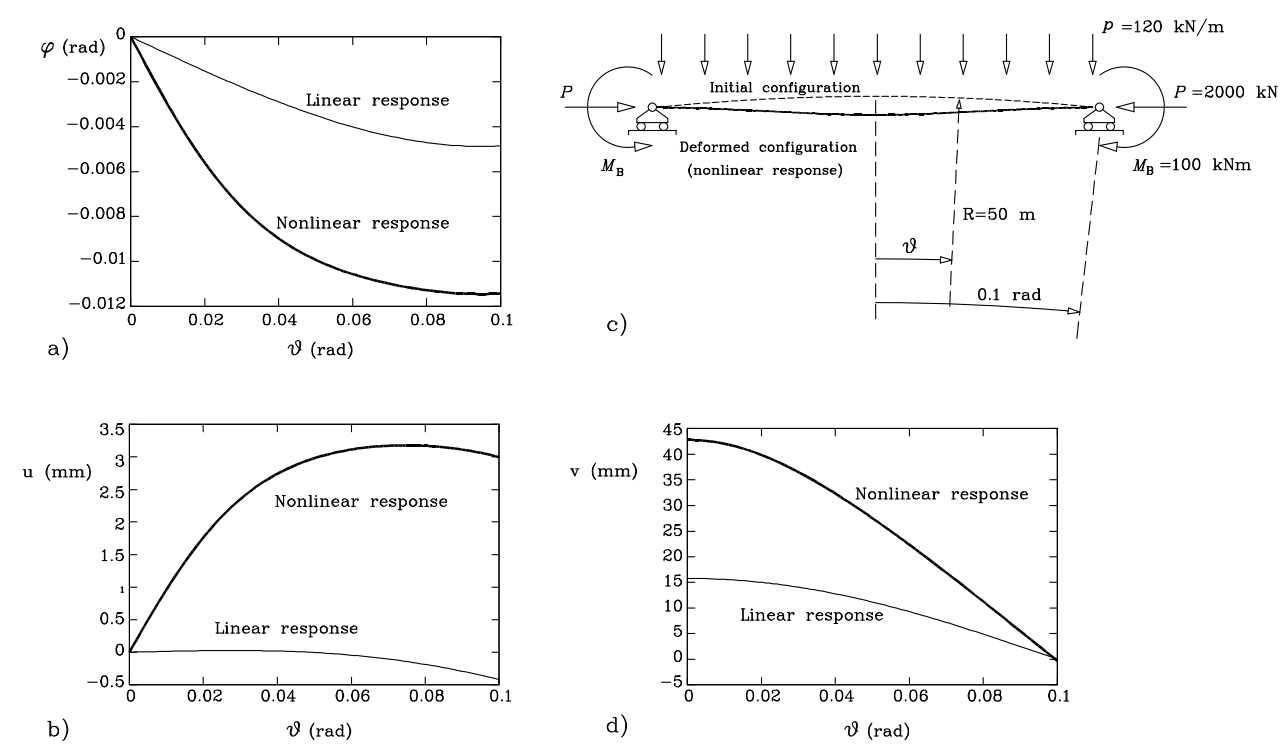

Figure 16. Rotation (a) and axial and transverse displacements (b, d) of the right side of the line of axis; (c) deformed shape, displacement magnified 10 times.

The evolution of the reactive forces and the distribution of the arch regions that behave nonlinearly can be followed for increasing loads. Moreover, a conventional value of the limit load based on the magnitude of the displacement at the keystone can be proposed. The proposed solution method described here can be readily extended to statically indeterminate problems. Such an extension is illustrated in a forthcoming paper by the authors (Aita et al., 2015b).

\subsection{Pointed, circular and elliptical masonry arches bearing vertical walls}

Let us consider a masonry arch subjected to its own weight and the weight of a superimposed wall (Figure 17). Some interesting approximate solutions to this equilibrium problem can be obtained by making some simplifying assumptions and limiting the treatment to some cases deemed particularly significant. In particular, we assume that the superimposed wall can be divided into vertical strips, the weight of each strip being sustained directly by the underlying arch element. Such a hypothesis is commonly adopted in technical practice and can be considered conservative in terms of safety. 

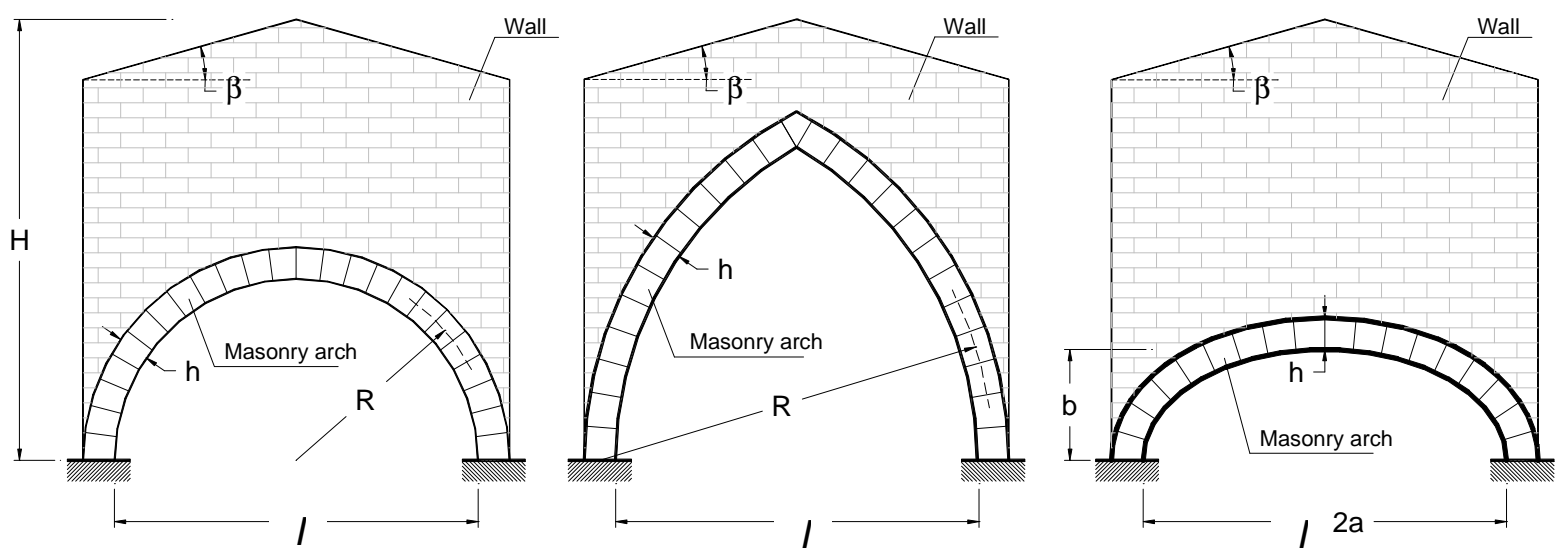

Figure 17. Scheme of the arch-wall system for circular (left), pointed (center) and elliptical (right) arches.

The first aspect we focus on concerns the influence of the masonry's limited compression strength on the arch's bearing capacity. The "classical" results of limit analysis under the Heyman hypotheses (1966) can in effect be easily compared with those obtained by applying the Durand-Claye method (1867), modified as described in section 2 in order to account for a nonlinear stress distribution both in tension and compression. For the sake of simplicity, in this first application of the Durand-Claye method, we assume the arch shear strength to be unbounded. Since the arches considered here are sufficiently thin, we assume that the influence of shear forces on the solution may be disregarded.

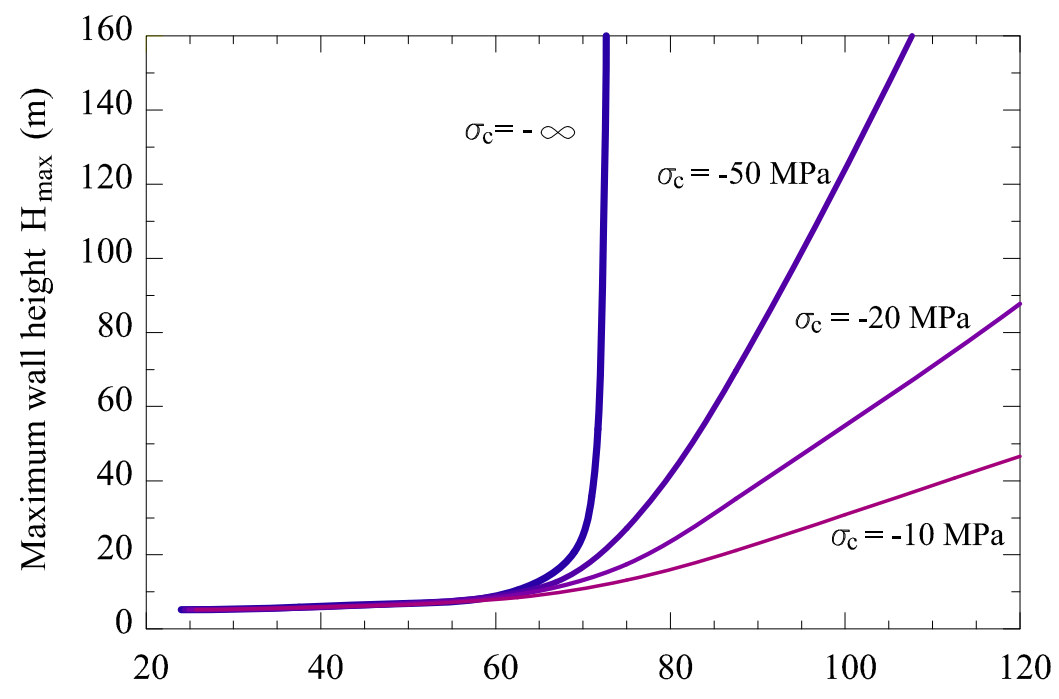

Arch thickness $h(\mathrm{~cm})$

Figure 18. Maximum height of the wall vs. arch thickness for different values of the masonry compressive strength (circular arch, $l=10 \mathrm{~m}$ ).

The stability area method allows for assessing the maximum height, $\mathrm{H}_{\max }$, of the overlying vertical wall that a masonry arch, assumed here to be circular, could sustain under equilibrium conditions as a function of both the arch thickness, $h$, and the masonry compressive strength, $\sigma_{c}$. Figure 18 shows the results obtained in terms of $\mathrm{H}_{\max }$ values as a function of the arch thickness 
for different constant $\sigma_{c}$ values. The effect of the limited compressive strength is evident and, what is more, turns out to be clearly non-negligible even for values of arch thickness and masonry compressive strength that are likely to be encountered in historical masonry structures. As expected, by taking $\sigma_{c}$ to be infinite, we revert to the well-known results obtained by Heyman through limit analysis. Analogous sets of results, omitted here for the sake of brevity, are obtained for pointed and elliptical arches as well.

The second aspect under examination concerns the mechanical response of the masonry arch to growing loads. The stability area method can then be applied to studying various arch-wall systems with different shapes. In all cases examined, some parameters were maintained constant: the clear span $l=10 \mathrm{~m}$; the compressive strength $\sigma_{c}=-20 \mathrm{MPa}$; the tensile strength $\sigma_{t}=0$. These values have been chosen from among the many possible alternatives in order to represent masonry of medium strength (good brick or sandstone masonry).

Once the problem has been defined in terms of its geometrical and mechanical aspects, it is a relatively simple matter to determine the maximum value of the wall height measured from the springings, $\mathrm{H}_{\max }$, corresponding to a limit condition for the arch-wall system. The results illustrated in the following figures have been obtained by means of an in-house, expressly developed algorithm implemented in Mathematica.

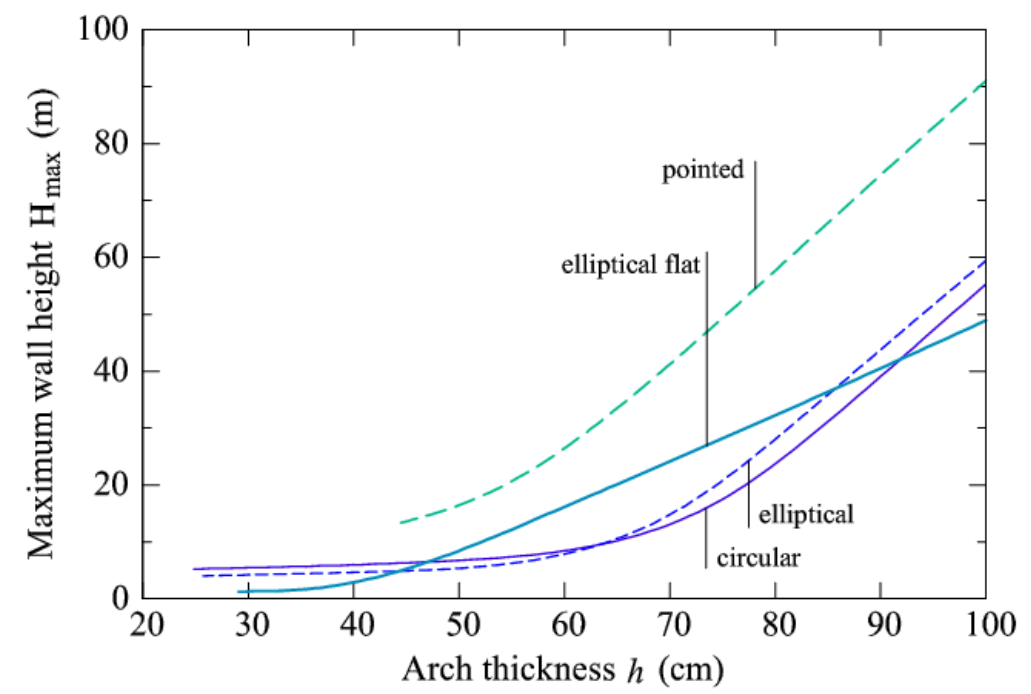

Figure 19. Maximum height of the wall vs. arch thickness for circular, elliptical and pointed arches $\left(l=10 \mathrm{~m}, \sigma_{c}=-20 \mathrm{MPa}\right)$.

Figure 19 sums up the most significant results obtained through the analyses. It shows a plot of the maximum height of the overlying wall for semicircular, elliptical and pointed arches. It is noteworthy that for any arch thickness, $h$, pointed arches allow for much greater wall heights than circular and elliptical ones. From an architectural point of view, such a result finds evident corroboration in the characteristically great heights attained by slender gothic structures, in which pointed arches are widely employed.

It is also rather interesting to note that for many values of $h$ commonly used in construction, elliptical flat arches also afford higher load-bearing capacities than circular arches of the same thickness. Such outstanding performance of elliptical flat arches can easily be attributed to their shape, which, in the presence of compressive stresses compatible with the limited material strength, evidentially manages to maintain the line of thrust within the arch thickness for values of $\mathrm{H}_{\max }$ that are decidedly higher than those attainable with circular and elliptical arches. 
The analyses conducted via the stability area method were aimed at evaluating the maximum height of the wall beyond which no equilibrium is possible for the arch in its initial configuration, under the hypothesis of limited masonry compressive strength. It cannot however be excluded that, as the situation approaches impending collapse and the structure's stiffness falls significantly, displacements will grow steadily, often accompanied by the emergence of extensive cracking. Thus, the geometric and constitutive nonlinearities could considerably degrade the actual ultimate load. Accounting for such aspects requires estimates of both the displacement and strain fields.

The evolution of the displacement, strain and stress fields with increasing external loads has been followed by making use of the simple one-dimensional nonlinear elastic model described in (Aita, Barsotti and Bennati, 2012). The nonlinear elastic analysis has been applied to study the four arch-wall systems previously investigated via the stability area method. The same main parameter values as before have been adopted. The clear span $l$ was maintained constant at $10 \mathrm{~m}$, compressive strength $\sigma_{c}$ at $-20 \mathrm{MPa}$, and tensile strength $\sigma_{t}$ at 0 ; lastly, a mean value of $4 \mathrm{GPa}$ was chosen for Young's modulus, $E$. The maximum attainable value of the wall height, $\mathrm{H}_{\max }$, under equilibrium conditions, once again measured from the springings and clearly dependent on the arch thickness, $h$, is defined as the maximum wall height value for which the numerical procedure results to be convergent (that is, in correspondence to which the error associated with the last iteration falls below the pre-set threshold within the maximum permitted number of iterations, here set equal to 1,000).

In order to compare the results of the nonlinear elastic analysis with those obtained through application of the Durand-Claye method, the springings of all the arches studied are assumed to be perfectly clamped. We moreover exclude the presence of any possible initial self-equilibrated stress fields, such as those caused, for instance, by settling at the abutments. Without attempting to delve further into the associated issues, we simply recall here the widely known fact that any settling or initial self-equilibrated stress field would not in any event influence the value of the collapse load.

Figure 20 shows the deformed lines of axis of the four different arches corresponding to the maximum wall heights (because of symmetry, only half arches are shown). The displacements near collapse may be so great as to cast serious doubts on the judiciousness of the commonly adopted choice of writing the equilibrium equations in the undeformed configuration.
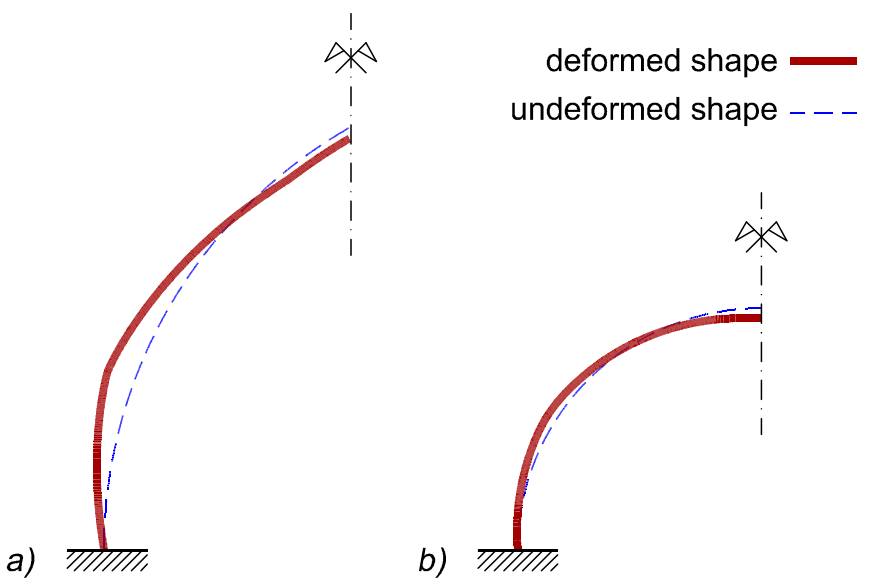

c)

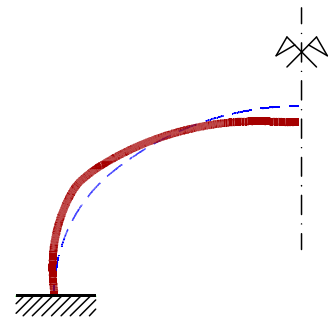

d)

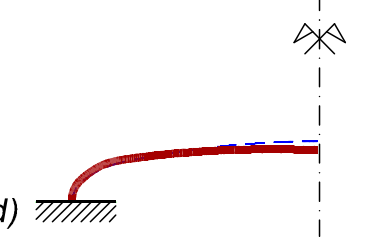

Figure 20. Deformed configurations of the arches close to collapse:

a) pointed; b) circular; c) elliptical; d) elliptical flat. 
As a consequence, when seeking to accurately evaluate the residual stiffness of the arch as it approaches collapse conditions, the geometrical nonlinearities stemming from the large displacements should probably be taken into careful consideration.

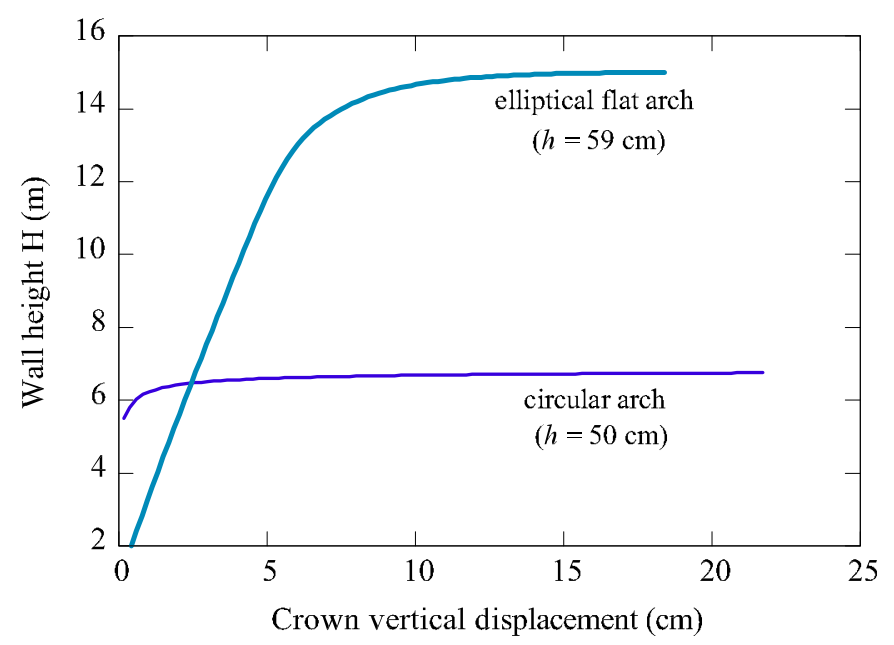

Figure 21. Wall height vs. crown vertical displacement (circular and elliptical flat arches, $\left.l=10 \mathrm{~m}, \sigma_{c}=-20 \mathrm{MPa}\right)$.

As the height of the wall grows, the nonlinear regions, under both tension and compression, become larger and larger. This will lower the overall arch stiffness, which could thus become very small relative to its initial value. The decrease in the stiffness of the arch is evident in Figure 21 , where the vertical displacements measured at the crown section are plotted against the wall height values, as measured from the springings. In particular, a $50 \mathrm{~cm}$ thick circular arch and a 59 $\mathrm{cm}$ thick elliptic flat arch are considered. The rapidly increasing growth in the displacements as the wall height reaches its limit value is noteworthy.

The behavior of the elliptical flat arch exhibits a wide linear elastic range, consistent with expectations that, as the height of the wall increases, the distribution of the nonlinear regions in tension remains nearly unchanged, and nonlinear behavior would emerge under compression only when the normal compressive strains become very large. Moreover, when linear behavior ceases, the arch is still capable of sustaining considerable increases in the height of the overlying wall. Instead, the circular arch rapidly loses its linear behavior and the subsequent collapse is not heralded by any perceptible nonlinear increases in load. Very good agreement is observed between the limit load values determined through the nonlinear elastic analysis and the modified Durand-Claye method.

\section{Concluding remarks}

The paper has addressed some equilibrium problems for masonry arches subject to vertical inplane loads. The solutions are pursued by means of suitable simplified schemes developed within two different theoretical frameworks: more precisely, a parallel study of masonry arches is performed via both limit and nonlinear elastic analyses.

The first solution technique is based on an expressly developed extension of the historical method of "stability areas" introduced by Durand-Claye in 1867. Our aim in modifying the method is to determine the set of statically admissible solutions within the limits imposed by the ultimate compressive and tensile strengths and the limited shear capacity of the joints. The second solution technique, instead, focuses on the stress and strain fields generated in the arch. Masonry's mechanical behavior, usually characterized by very low resistance to tension, is 
represented, as a first approximation, via a nonlinear elastic constitutive relation incorporated into a one-dimensional model.

In the present work, the two different solution techniques have been applied to three problems. Each of the first two applications has focused on one of the two solution methods to highlight its main features. In particular, the first case describes an example application of the stability areas method to the collapse of pointed arches by accounting for masonry's limited shear strength. Among other things, the method has enabled determining the range of values of the arch thickness and the masonry friction coefficient that correspond to each different sliding collapse mode.

The second case demonstrates that nonlinear elastic analysis enables obtaining the explicit solution, in terms of displacements, stresses and extension of nonlinear regions, where masonry cracking and damage are to be expected. It regards some particular cases, such as that of depressed arches subjected to uniformly distributed vertical loads, and calls for making some reasonable approximations. However, the results obtained suggest that the search for the solution to more general cases could be much more demanding, and is therefore likely to necessitate recourse to suitable numerical techniques.

The last problem, concerning a mechanical system composed of an arch and overlying wall, serves to illustrate combined application of the two methods. The problem considered - actually quite a common one in masonry buildings and bridges - has been solved for arches of different shapes, namely: pointed, circular and elliptical. The results obtained confirmed the well-known fact that shape strongly affects the load capacity of a masonry arch and clearly indicate that pointed arches are expected to exhibit better performances than circular and elliptical ones.

The solved examples highlight that the two methods perform complementary functions: the stability area method allows for readily determining a collapse load value, while the nonlinear elastic analysis provides a helpful and, in some aspects, essential check of its mechanical significance by following the evolution of the displacement field and extension of the nonlinear regions where cracking and crushing phenomena arise as the load increases.

In conclusion, the results reported in the present paper suggest that by simultaneously using the two solution methods it is possible, on the one hand, to readily determine the collapse load by accounting for both the joint's limited shear strength and masonry's limited compressive strength, and, on the other, to reconstruct a concise, yet often comprehensive, description of the evolution of the displacement and stress fields within an arch subject to in-plane increasing loads. In particular, the models presented here seem able to provide useful indications in all those cases in which the actual conditions or the conservation state of existing masonry arches should be assessed, or when planning restoration operations.

\section{REFERENCES}

AITA D., BARSOTTI R., BENNATI S. 2003. Some explicit solutions for flat and depressed masonry arches. In: Proceedings of the first international congress on construction history (Madrid, $20^{\text {th }}-24^{\text {th }}$ January 2003). Vol. I. Madrid: Instituto Juan De Herrera. 171-183.

AitA D., BARSOTTI R., BENNATI S., FOCE F. 2003. Soluzioni esplicite per l'analisi elastica non lineare e l'analisi limite di strutture ad arco in muratura. In: Atti del XVI Congresso AIMETA di meccanica teorica e applicata (Ferrara, 9-12 settembre 2003). Memoria: su CD rom. Sommario: 149.

AITA D., BARSOTTI R., BENNATI S. AND FOCE F. 2004. The statics of pointed masonry arches between 'limit' and 'elastic' analysis. In: Arch Bridges IV. Advances in Assessment, Structural Design and Construction. Edited by P. Roca, C. Molins. Barcelona: CIMNE. 354-362. 
AitA D., BARSOTTI R., BENNATI S. AND FocE F. 2007. Collapse of masonry arches in Romanesque and Gothic constructions. In: ARCH'07, Proceedings of 5th International Conference on Arch Bridges (Madeira, 12-14 September 2007). Edited by P. B. Lourenço, D. V. Oliveira, A. Portela. Guimarães (Portugal): University of Minho. 625-32.

AitA D., BARSOTTI R. AND BENNATI S. 2009. Load-bearing capacity of circular, pointed and elliptical masonry arches. In: Aimeta 2009. Atti del $19^{\circ}$ Congresso dell'Associazione italiana di meccanica teorica e applicata (Ancona, 14-17 settembre 2009). Ancona: Aras Edizioni.

AITA D., BARSOTTI R., BENNATI S. 2012. Equilibrium of pointed, circular and elliptical masonry arches bearing vertical walls. Journal of Structural Engineering. ASCE.

Aita D., BARsotTi R., Bennati S. 2015. Explicit Solutions for Depressed Masonry Arches Loaded until Collapse - Part I: the Mechanical Model. Submitted to Meccanica.

Aita D., BARsotTi R., BenNati S. 2015. Explicit Solutions for Depressed Masonry Arches Loaded until Collapse - Part II: Applications and Results. Submitted to Meccanica.

Alfano G., Rosati L. AND Valoroso N. 2000. A numerical strategy for finite element analysis of notension materials. Int. J. Numer. Meth. Engng, 48. 317-50.

Baggio C. AND TROVAlusCi P. 1988. Limit analysis for no-tension and frictional three-dimensional discrete systems. Mechanics of Structures and Machines, 26, 1998. 287-304.

Baggio C. AND Trovalusci P. 2000. Collapse behaviour of three-dimensional brick-block systems using non-linear programming. Structtural Engineering and Mechanics, 10(2), 2000. 181-195.

BARSOTTI R. AND BENNATI S. 2002. Non-linear analysis and collapse of masonry arches. In: Becchi A., Corradi M., Foce F., Pedemonte O. (eds.), Towards a history of construction. Dedicated to Edoardo Benvenuto, series Between mechanics and architecture. Basel: Birkhäuser. 53-71.

BENNATI S. AND DAMI A. 2009. A simple structural model for a masonry arch-wall system subjected to dead vertical loads. In: Aimeta 2009. Atti del $19^{\circ}$ Congresso dell'Associazione italiana di meccanica teorica e applicata (Ancona, 14-17 settembre 2009). Ancona: Aras Edizioni.

BENNATI S. AND BARSOTTI R. 1999. Comportamento non lineare di archi in muratura. In: Atti del XIV Congresso Nazionale AIMETA (Como, 6-9 ottobre 1999).

BENNATI S. AND BARSOTTI R. 2001. Optimum radii of circular masonry arches. In: ARCH'01, Third international arch bridges conference (Paris, 19-21 september 2001), vol. 1. Edited by C. Abdunur. Paris: Presses de l'école nationale des ponts et chaussées. 489-98.

CAVICCHI A. AND GAMBAROTTA L. 2005. Collapse analysis of masonry bridges taking into account archfill interaction. Engineering Structures, 27. 605-15.

DURAND-CLAYE A. 1867. Note sur la vérification de la stabilité des voûtes en maçonnerie et sur l'emploi des courbes de pression. Ann. des Ponts et Chaussées, 13. 63-93.

FOCE F. AND AITA D. 2003. The masonry arch between 'limit' and 'elastic' analysis. A critical reexamination of Durand-Claye's method. In: Proceedings of the first international congress on construction history (Madrid, $20^{\text {th }}-24^{\text {th }}$ January 2003), vol. 2. Edited by S. Huerta. Madrid: Instituto Juan De Herrera. 895-908.

HeYMAn J. 1966. The stone skeleton. Int. J. Solids Structures, 2. 249-79.

HEYMAN J. 1969. The safety of masonry arches. Int. Journal of Mechanical Sciences. 11. 363-385.

LOURENÇO P.B. 2005. Assessment, diagnosis and strengthening of Outeiro Church, Portugal. Construction and Building Materials, 19. 634-45.

SIGNORINI A. 1925a. Un teorema di esistenza e unicità nella statica dei materiali poco resistenti a trazione, In: Rendiconti dell'Accademia dei Lincei, Serie VI, II. 401-406.

SigNORINI A. 1925b. Sulla pressoflessione delle murature. In: Rendiconti dell'Accademia dei Lincei, Serie VI, II. 484-489.

SINOPOLI A., AITA D., FOCE F., 2007. Further remarks on the collapse of masonry arches with Coulomb friction. In: ARCH'07, Proceedings of 5th International Conference on Arch Bridges (Madeira, 12-14 
September 2007), edited by P. B. Lourenço, D. V. Oliveira, A. Portela. Guimarães (Portugal): University of Minho. 649-657. 